\title{
Evaluation of the electric force in electrophoresis
}

\author{
Jyh-Ping Hsu *, Li-Hsien Yeh, Ming-Hong Ku \\ Department of Chemical Engineering, National Taiwan University, Taipei, Taiwan 10617
}

Received 24 August 2006; accepted 29 September 2006

Available online 30 October 2006

\begin{abstract}
A new expression for the evaluation of the electric force acting on a colloidal particle in an applied electric field is derived under the condition of weak applied electric field. The expression derived, which is based on the Maxwell stress tensor, is applicable to both rigid and soft particles for various types of surface conditions and to both symmetric and asymmetric geometries. We show that, depending upon the electrophoresis conditions, the electric force evaluated by the methods commonly used in the literature can be overestimated, thereby leading to incorrect electrophoretic mobility.
\end{abstract}

(c) 2006 Elsevier Inc. All rights reserved.

Keywords: Electrophoretic mobility; Maxwell stress tensor; Various types of charged conditions

\section{Introduction}

Electrophoresis is used widely by experimentalists in various fields to characterize the charged conditions on the surface of an entity of colloidal size [1,2]. It is also frequently used as a tool to separate entities of similar physical properties that cannot be differentiated by other typical analytical methods [3,4]. The most important output of electrophoresis measurement is the electrophoretic mobility, defined as the electrophoretic velocity per unit strength of an applied electric field. Theoretically, this quantity is determined from the fact that the total force, which includes the electrical force and the hydrodynamic force, acting on an entity vanishes in the steady state. Several different expressions have been used to evaluate the latter in various types of problems. These include, for example, problems of total symmetric nature such as an isolated sphere in an infinite medium [5] or a dispersion of spherical particles [6,7], and those not of total symmetric nature such as two spheres in an infinite medium [8]. Often, because detailed derivations of these expressions are not presented, their applicability needs justification.

The purpose of this work is to derive, in detail, the appropriate expressions to evaluate the electrical force acting on an

\footnotetext{
* Corresponding author. Fax: +8862 23623040.

E-mail address: jphsu@ntu.edu.tw (J.-P. Hsu).
}

entity under conditions of practical significance. The applicability of the relevant expressions in the literature is also discussed.

\section{Theory}

Let us consider the general case illustrated in Fig. 1, where a colloidal particle of surface $\Omega_{\mathrm{p}}$ is placed in a medium, which may have a boundary of surface $\Omega_{\mathrm{b}}$. The space between the particle and the boundary is filled with an aqueous solution

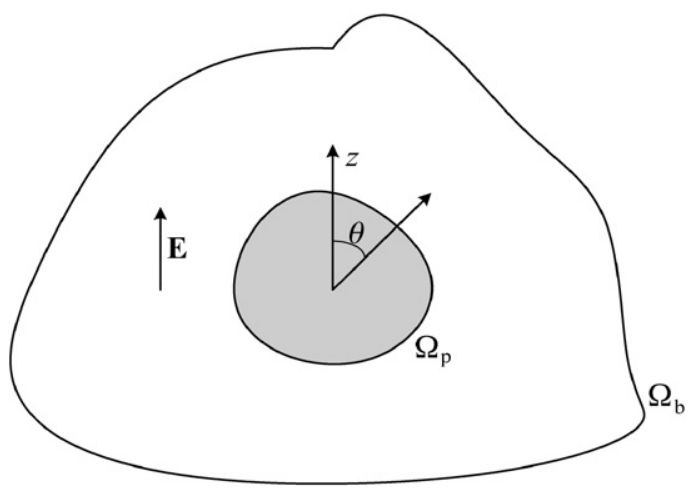

Fig. 1. Schematic representation of the problem considered where a colloidal particle of surface $\Omega_{\mathrm{p}}$ is placed in a medium, which may have a boundary of surface $\Omega_{\mathrm{b}}$. A uniform electric field $\mathbf{E}$ parallel to the $z$-direction is applied; $\theta$ is the solid angle. 
containing $z_{1}: z_{2}$ electrolytes; $z_{1}$ and $z_{2}$ are, respectively, the valences of cations and anions. A uniform electric field $\mathbf{E}$ of strength $E$ is applied in the $z$-direction. We assume that the liquid phase is an incompressible Newtonian fluid. The applied electric field is relatively weak compared with that established by a charged surface. This is realistic, since the surface potential is on the order of $25.7 \mathrm{mV}$ to $10 \mathrm{kV}$ and the Debye length ranges from $10 \mathrm{~nm}$ to $1 \mu \mathrm{m}$, which implies that the strength of the electric field established ranges from 25.7 to $10^{9} \mathrm{kV} / \mathrm{m}$. The strength of the applied electric field in practice is much lower than these values. Both the surface of the particle and that of the boundary are nonconductive and nonslip.

\subsection{Electrokinetic equations}

Since the Reynolds number in electrophoresis is very small, the governing equations for the present problem can be expressed as

$\nabla \cdot\left[n_{j} \mathbf{u}-D_{j}\left(\nabla n_{j}+\frac{z_{j} e n_{j}}{k_{\mathrm{B}} T} \nabla \Psi\right)\right]=0$,

$\nabla^{2} \Psi=-\frac{\rho_{e}}{\varepsilon}=-\sum_{j=1}^{2} \frac{z_{j} e n_{j}}{\varepsilon}$,

$\nabla \cdot \mathbf{u}=0$

$0=\eta \nabla^{2} \mathbf{u}-\nabla p+\rho_{e} \mathbf{E}$.

In these expressions $\nabla$ and $\nabla^{2}$ are, respectively, the gradient operator and the Laplace operator, $D_{j}, n_{j}$, and $z_{j}$ are, respectively, the diffusion coefficient, the number concentration, and the valence of ionic species $j, e$ is the elementary charge, $k_{\mathrm{B}}$ is the Boltzmann constant, $T$ is the absolute temperature, $\Psi$ is the electric potential, $\varepsilon$ is the permittivity of the liquid phase, $\rho_{e}$ is the space charge density, and $\mathbf{u}, \eta$, and $p$ are, respectively, the velocity, the viscosity, and the pressure of the liquid phase. $\mathbf{E}=-\nabla \Psi$ and $\rho_{e} \mathbf{E}$ is the electric body force acting on the liquid.

If the applied electric field is weak, $\mathbf{u}, p, \Psi$, and $n_{j}$ can be expressed, respectively, as $[9,10]$

$\mathbf{u}=\mathbf{u}^{(0)}+\delta \mathbf{u}$

$p=p^{(0)}+\delta p$

$\Psi=\Psi^{(0)}+\delta \Psi$,

$n_{j}=n_{j}^{(0)}+\delta n_{j}$.

Here, a quantity with superscript (0) refers to the equilibrium value; that is, when $\mathbf{E}$ is not applied, a quantity with prefix $\delta$ denotes a perturbed value arising from $\mathbf{E}$. Note that $\mathbf{u}^{(0)}=0$. By substituting Eqs. (5)-(8) into Eqs. (1)-(4), the problem under consideration can be divided into an equilibrium problem and a perturbed problem.

\subsubsection{Equilibrium problem}

Let us consider first the quantities in the equilibrium state. For the electrical field, we have

$\nabla n_{j}^{(0)}+\frac{z_{j} e n_{j}^{(0)}}{k_{\mathrm{B}} T} \nabla \Psi^{(0)}=0$, $\nabla^{2} \Psi^{(0)}=-\sum_{j=1}^{2} \frac{z_{j} e n_{j}^{(0)}}{\varepsilon}$,

$0=-\nabla p^{(0)}+\varepsilon \nabla^{2} \Psi^{(0)} \nabla \Psi^{(0)}$

Integrating Eq. (9) from $\Psi^{(0)}=0$ and $n_{j}^{(0)}=n_{j}^{\infty}$ to $\Psi^{(0)}=\Psi^{(0)}$ and $n_{j}^{(0)}=n_{j}^{(0)}$ leads to a Boltzmann distribution

$n_{j}^{(0)}=n_{j}^{\infty} \exp \left(-\frac{z_{j} e \Psi^{(0)}}{k_{\mathrm{B}} T}\right)$,

where $n_{j}^{\infty}$ is the bulk concentration of ionic species $j$. Substituting Eq. (12) into Eq. (10) gives

$\nabla^{2} \Psi^{(0)}=-\sum_{j=1}^{2} \frac{z_{j} e n_{j}^{\infty}}{\varepsilon} \exp \left(\frac{z_{j} e \Psi^{(0)}}{k_{\mathrm{B}} T}\right)$.

Combining Eqs. (10) and (11) yields

$-\nabla p^{(0)}=\varepsilon \sum_{j=1}^{2} \frac{z_{j} e n_{j}^{(0)}}{\varepsilon} \nabla \Psi^{(0)}$.

Integrating this expression from $p^{(0)}=p^{\infty}$ and $n_{j}^{(0)}=n_{j}^{\infty}$ to $p^{(0)}=p^{(0)}$ and $n_{j}^{(0)}=n_{j}^{(0)}$ yields

$p^{(0)}=p^{\infty}+\sum_{j=1}^{2} k_{\mathrm{B}} T\left(n_{j}^{(0)}-n_{j}^{\infty}\right)$.

This is the equilibrium or static pressure distribution, where $\mathbf{u}=\mathbf{0}$.

\subsubsection{Perturbed problem}

Under the condition of weak applied electric field, O'Brien and White [9] proposed using the expression

$n_{j}=n_{j}^{\infty} \exp \left(-\frac{z_{j} e\left(\Psi^{(0)}+\delta \Psi+g_{j}\right)}{k_{\mathrm{B}} T}\right), \quad j=1,2$,

to take the effect of double-layer polarization into account, where $g_{j}$ is a potential used to describe double-layer polarization. The governing equation for $\delta \Psi$ can be obtained from Eqs. (13) and (16), and $\Psi=\Psi^{(0)}+\delta \Psi$ as

$$
\begin{aligned}
\nabla^{2} \delta \Psi= & \nabla^{2} \Psi-\nabla^{2} \Psi^{(0)} \\
= & -\sum_{j=1}^{2} \frac{z_{j} e n_{j}^{\infty}}{\varepsilon}\left[\exp \left(-\frac{z_{j} e\left(\Psi^{(0)}+\delta \Psi+g_{j}\right)}{k_{\mathrm{B}} T}\right)\right. \\
& \left.-\exp \left(\frac{z_{j} e \Psi^{(0)}}{k_{\mathrm{B}} T}\right)\right] .
\end{aligned}
$$

Combining Eqs. (8), (12), and (16) yields

$$
\begin{aligned}
\delta n_{j}= & n_{j}^{\infty} \exp \left(-\frac{z_{j} e\left(\Psi^{(0)}+\delta \Psi+g_{j}\right)}{k_{\mathrm{B}} T}\right) \\
& -n_{j}^{\infty} \exp \left(-\frac{z_{j} e \Psi^{(0)}}{k_{\mathrm{B}} T}\right) .
\end{aligned}
$$

Substituting Eqs. (5), (7), and (18) into Eq. (1), we obtain 


$$
\begin{aligned}
& \nabla^{2} g_{j}-\frac{z_{j} e}{k_{\mathrm{B}} T} \nabla \Psi^{(0)} \cdot \nabla g_{j}=\frac{1}{D_{j}} \delta \mathbf{u} \cdot \nabla \Psi+\frac{1}{D_{j}} \delta \mathbf{u} \cdot \nabla g_{j} \\
& \quad+\frac{z_{j} e}{k_{\mathrm{B}} T} \nabla \delta \Psi \cdot \nabla g_{j}+\frac{z_{j} e}{k_{\mathrm{B}} T} \nabla g_{j} \cdot \nabla g_{j} .
\end{aligned}
$$

Because $\delta \mathbf{u}, \delta p, \delta \Psi$, and $\delta n_{j}$ are all on the order of $E$, the products of any two of these quantities becomes negligible. Therefore, the governing equations for the flow field, by collecting terms on the order of $E$ only, are

$\nabla \cdot \delta \mathbf{u}=0$

$0=\eta \nabla^{2} \delta \mathbf{u}-\nabla \delta p+\varepsilon \nabla^{2} \Psi^{(0)} \nabla \delta \Psi+\varepsilon \nabla^{2} \delta \Psi \nabla \Psi^{(0)}$.

For convenience, $\Psi^{(0)}, \delta \Psi, \delta \mathbf{u}$, and $\delta p$ are replaced, respectively, by $\Psi_{1}, \Psi_{2}$, u, and $p$, and Eqs. (13), (17), and (19)-(21) are rewritten as

$$
\begin{aligned}
& \nabla^{2} \Psi_{1}=-\sum_{j=1}^{2} \frac{z_{j} e n_{j}^{\infty}}{\varepsilon} \exp \left(\frac{z_{j} e \Psi_{1}}{k_{\mathrm{B}} T}\right), \\
& \nabla^{2} \Psi_{2}=-\sum_{j=1}^{2} \frac{z_{j} e n_{j}^{\infty}}{\varepsilon} \\
& \times\left[\exp \left(-\frac{z_{j} e\left(\Psi_{1}+\Psi_{2}+g_{j}\right)}{k_{\mathrm{B}} T}\right)-\exp \left(\frac{z_{j} e \Psi_{1}}{k_{\mathrm{B}} T}\right)\right], \\
& \nabla^{2} g_{j}-\frac{z_{j} e}{k_{B} T} \nabla \Psi_{1} \cdot \nabla g_{j}=\frac{1}{D_{j}} \mathbf{u} \cdot \nabla \Psi+\frac{1}{D_{j}} \mathbf{u} \cdot \nabla g_{j} \\
& +\frac{z_{j} e}{k_{\mathrm{B}} T} \nabla \Psi_{2} \cdot \nabla g_{j}+\frac{z_{j} e}{k_{\mathrm{B}} T} \nabla g_{j} \cdot \nabla g_{j}, \\
& \nabla \cdot \mathbf{u}=0, \\
& 0=\eta \nabla^{2} \mathbf{u}-\nabla p+\varepsilon \nabla^{2} \Psi_{1} \nabla \Psi_{2}+\varepsilon \nabla^{2} \Psi_{2} \nabla \Psi_{1} .
\end{aligned}
$$

In the last expression, the sum of the last two terms on its righthand side denotes the electric body force acting on the liquid.

\subsubsection{Low surface potential}

The special case of low surface potential is often discussed in the literature. In this case the effect of double-layer polarization can be neglected, $\delta n_{j}=0$ [11], and Eqs. (22)-(26) become [1215]

$$
\begin{aligned}
& \nabla^{2} \Psi_{1}=\kappa^{2} \Psi_{1}, \\
& \nabla^{2} \Psi_{2}=0, \\
& \nabla \cdot \mathbf{u}=0, \\
& 0=\eta \nabla^{2} \mathbf{u}-\nabla p+\varepsilon \nabla^{2} \Psi_{1} \nabla \Psi_{2} .
\end{aligned}
$$

The last term on the right-hand side of Eq. (30) denotes the electric body force, where $-\varepsilon \nabla^{2} \Psi_{1}=\rho_{e}$ is the space charge density and $-\nabla \Psi_{2}=\mathbf{E}$ is the applied electric field [12-15]. In the literature, the electric body force is represented by $-\rho_{e} \nabla \Psi=$ $\varepsilon \nabla^{2} \Psi \nabla \Psi$ in many studies $[6,7,10,16-25]$, some of which are not of total symmetric nature [16,21,22,24,25]. That is, Eq. (26) or Eq. (30) is replaced by

$0=\eta \nabla^{2} \mathbf{u}-\nabla p-\varepsilon \nabla^{2} \Psi \nabla \Psi$.

Note that for a system not of total symmetric nature, such as a sphere normal to a plane [22,25], an extraneous electrostatic force $\varepsilon \nabla^{2} \Psi_{1} \nabla \Psi_{1}$ is included in Eq. (31). This implies that the electric field established by the equilibrium electric potential, $-\nabla \Psi_{1}$, is also a driving force for the electrophoresis of a particle and the mobility will be overestimated.

\subsection{Scaled governing equations}

For a more concise treatment, the scaled quantities are used in subsequent analyses. To this end, the following scaling factors are chosen respectively for the length scale, the electrical potential, the concentration of ionic species, and the velocity: the radius of a particle a, the equilibrium surface potential $\zeta_{\mathrm{a}}$, the bulk concentration of electrolyte $n_{j}^{\infty}$, and the reference electrophoretic velocity $U_{\mathrm{E}}=\varepsilon \zeta_{\mathrm{a}}^{2} / \eta a$. In terms of scaled quantities, the governing equations for an arbitrary surface potential, Eqs. (22)-(26), can be rewritten as [25-28]

$$
\begin{aligned}
& \nabla^{* 2} \Psi_{1}^{*}=-\frac{1}{(1+\alpha)} \frac{(\kappa a)^{2}}{\Psi_{\mathrm{r}}}\left[\exp \left(-\Psi_{\mathrm{r}} \Psi_{1}^{*}\right)-\exp \left(\alpha \Psi_{\mathrm{r}} \Psi_{1}^{*}\right)\right] \\
& \nabla^{* 2} \Psi_{2}^{*}-\frac{(\kappa a)^{2}}{(1+\alpha)}\left[\exp \left(-\Psi_{\mathrm{r}} \Psi_{1}^{*}\right)+\alpha \exp \left(\alpha \Psi_{\mathrm{r}} \Psi_{1}^{*}\right)\right] \Psi_{2}^{*} \\
& \quad=\frac{(\kappa a)^{2}}{(1+\alpha)}\left[\exp \left(-\Psi_{\mathrm{r}} \Psi_{1}^{*}\right) g_{1}^{*}+\exp \left(\alpha \Psi_{\mathrm{r}} \Psi_{1}^{*}\right) \alpha g_{2}^{*}\right] \\
& \nabla^{* 2} g_{1}^{*}-\Psi_{\mathrm{r}} \nabla^{*} \Psi_{1}^{*} \cdot \nabla^{*} g_{1}^{*}=\Psi_{\mathrm{r}}^{2} \operatorname{Pe}_{1} \mathbf{u}^{*} \cdot \nabla^{*} \Psi_{1}^{*} \\
& \nabla^{* 2} g_{2}^{*}+\alpha \Psi_{\mathrm{r}} \nabla^{*} \Psi_{1}^{*} \cdot \nabla^{*} g_{2}^{*}=\Psi_{\mathrm{r}}^{2} \operatorname{Pe}_{2} \mathbf{u}^{*} \cdot \nabla^{*} \Psi_{1}^{*} \\
& \nabla^{*} \cdot \mathbf{u}^{*}=0 \\
& 0=\nabla^{* 2} \mathbf{u}^{*}-\nabla^{*} p^{*}+\nabla^{* 2} \Psi_{1}^{*} \nabla^{*} \Psi_{2}^{*}+\nabla^{* 2} \Psi_{2}^{*} \nabla^{*} \Psi_{1}^{*}
\end{aligned}
$$

where a symbol with an asterisk denotes a scaled quantity; $\Psi_{\mathrm{r}}=\zeta_{\mathrm{a}} /\left(z_{1} e / k_{\mathrm{B}} T\right)$ is the scaled surface potential of the particle; $\mathrm{Pe}_{j}=\varepsilon\left(z_{1} e / k_{\mathrm{B}} T\right)^{2} / \eta D_{j}, j=1,2$, is the electric Peclet number of ionic species $j ; \alpha=-z_{2} / z_{1}$; and $\kappa=\left[\sum_{j=1}^{2}\left(n_{j}^{\infty}\left(e z_{j}\right)^{2} / \varepsilon k_{\mathrm{B}} T\right)\right]^{1 / 2}$ is the reciprocal Debye length.

Similarly, the scaled governing equations for the case of low surface potential are

$\nabla^{* 2} \Psi_{1}^{*}=(\kappa a)^{2} \Psi_{1}^{*}$,

$\nabla^{* 2} \Psi_{2}^{*}=0$

$\nabla^{*} \cdot \mathbf{u}^{*}=0$

$0=\nabla^{* 2} \mathbf{u}^{*}-\nabla^{*} p^{*}+\nabla^{* 2} \Psi_{1}^{*} \nabla^{*} \Psi_{2}^{*}$.

\subsection{Boundary conditions}

\subsubsection{Electric field}

Three types of boundary conditions are usually assumed: constant surface potential $[8,12,13,16-20,25-29]$, constant surface charge density $[15,16,21]$, and charge-regulated surface [6,7,14,22-24]. If the surface potential of a particle and that of a boundary are maintained respectively at $\zeta_{\mathrm{a}}$ and $\zeta_{\mathrm{b}}$, then the boundary conditions for $\Psi_{1}$ and $\Psi_{2}$ are

$\Psi_{1}=\zeta_{\mathrm{a}} \quad$ on $\Omega_{\mathrm{p}}$,

$\Psi_{1}=\zeta_{\mathrm{b}} \quad$ on $\Omega_{\mathrm{b}}$,

$\mathbf{n} \cdot \nabla \Psi_{2}=0 \quad$ on $\Omega_{\mathrm{p}}$, 
n. $\nabla \Psi_{2}=-E \cos \theta$ on $\Omega_{\mathrm{b}}$,

where $\mathbf{n}$ is the unit normal vector directed into the liquid phase. If the surface of a particle and that of a boundary are maintained at constant surface charge density, then the corresponding boundary conditions become

$\mathbf{n} \cdot \nabla \Psi_{1}=-\frac{\sigma_{\mathrm{a}}}{\varepsilon} \quad$ on $\Omega_{\mathrm{p}}$,
$\mathbf{n} \cdot \nabla \Psi_{1}=-\frac{\sigma_{\mathrm{b}}}{\varepsilon} \quad$ on $\Omega_{\mathrm{b}}$,
$\mathbf{n} \cdot \nabla \Psi_{2}=0$ on $\Omega_{\mathrm{p}}$,
$\mathbf{n} \cdot \nabla \Psi_{2}=-E \cos \theta \quad$ on $\Omega_{\mathrm{b}}$,

. $\nabla \Psi_{2}=-E \cos \theta$ on $\Omega_{\mathrm{b}}$

where $\sigma_{\mathrm{a}}$ and $\sigma_{\mathrm{b}}$ are respectively the surface charge density of the particle and that of the boundary. For the case of a chargeregulated surface, let us consider, for example, the dissociation reaction below on the surface of a particle [7]:

$\mathrm{AH} \Leftrightarrow \mathrm{A}^{-}+\mathrm{H}^{+}$.

It can be shown that the charge density on the particle surface, $\sigma_{\mathrm{a}}$, is

$\sigma_{\mathrm{a}}=-e\left[A^{-}\right]=-\frac{e N_{\mathrm{S}}}{1+\left(\left[H^{+}\right]_{\mathrm{b}} / K_{\mathrm{a}}\right) \exp \left(-\left(e \Psi_{1} / k_{\mathrm{B}} T\right)\right)}$,

where $K_{\mathrm{a}}=\left[\mathrm{A}^{-}\right]_{\mathrm{S}}\left[\mathrm{H}^{+}\right]_{\mathrm{S}} /[\mathrm{AH}]_{\mathrm{S}}$ is the equilibrium dissociation constant for the dissociation reaction represented by Eq. (50), and $N_{\mathrm{S}}$ is the density of dissociable functional groups on the particle surface. Here, a symbol with square brackets denotes the concentration of a species; the subscripts $S$ and $b$ represent respectively the surface property and the bulk liquid property. In this case, the boundary condition associated with $\Psi_{1}$ on the particle surface, Eq. (46), needs to be replaced by

n. $\nabla \Psi_{1}=-\frac{e N_{\mathrm{S}} / \varepsilon}{1+\left(\left[\mathrm{H}^{+}\right]_{\mathrm{b}} / K_{\mathrm{a}}\right) \exp \left(-\left(e \Psi_{1} / k_{\mathrm{B}} T\right)\right)}$.

If we let $\nabla^{*}=a \nabla, \Psi_{1}^{*}=e \Psi_{1} / k_{\mathrm{B}} T, A=e^{2} N_{\mathrm{S}} a / \varepsilon k_{\mathrm{B}} T$, and $B=\left[\mathrm{H}^{+}\right]_{\mathrm{b}} / K_{\mathrm{a}}$, then this expression can be rewritten as

$\mathbf{n} \cdot \nabla^{*} \Psi_{1}^{*}=-\frac{A}{1+B \exp \left(-\Psi_{1}^{*}\right)}$.

If $\Psi_{1}$ is low, it can be shown that the corresponding surface charge density $\sigma_{\mathrm{a}}$ is $[6,14,22-24]$

$\sigma_{\mathrm{a}}=-\frac{e N_{\mathrm{S}}}{\left\{1+\left[\mathrm{H}^{+}\right]_{\mathrm{b}} / K_{\mathrm{a}}\right\}^{2}}-\frac{\left(e^{2} N_{\mathrm{S}} / k_{\mathrm{B}} T\right)\left\{\left[\mathrm{H}^{+}\right]_{\mathrm{b}} / K_{\mathrm{a}}\right\}}{\left\{1+\left[\mathrm{H}^{+}\right]_{\mathrm{b}} / K_{\mathrm{a}}\right\}^{2}} \Psi_{1}$.

In this case, Eq. (46) reduces to

$$
\begin{aligned}
\mathbf{n} \cdot \nabla \Psi_{1}= & -\frac{e N_{\mathrm{S}} / \varepsilon}{\left\{1+\left[\mathrm{H}^{+}\right]_{\mathrm{b}} / K_{\mathrm{a}}\right\}} \\
& -\frac{\left(e^{2} N_{\mathrm{S}} / \varepsilon k_{\mathrm{B}} T\right)\left\{\left[\mathrm{H}^{+}\right]_{\mathrm{b}} / K_{\mathrm{a}}\right\}}{\left\{1+\left[\mathrm{H}^{+}\right]_{\mathrm{b}} / K_{\mathrm{a}}\right\}^{2}} \Psi_{1},
\end{aligned}
$$

or in terms of scaled symbols as

$\mathbf{n} \cdot \nabla^{*} \Psi_{1}^{*}=\frac{A}{1+B}-\frac{A B}{[1+B]^{2}} \Psi_{1}^{*}$.

Suppose that the ionic concentration reaches the equilibrium value on the boundary surface and the surface of a particle is ion-impenetrable. Then the boundary conditions for $g_{j}$ are [9]

$g_{j}=-\Psi_{2} \quad$ on $\Omega_{\mathrm{b}}$,

$n \cdot \nabla g_{j}=0 \quad$ on $\Omega_{\mathrm{p}}$.

\subsubsection{Flow field}

If we let $U$ be the particle velocity in the $z$-direction and $\mathbf{e}_{z}$ be the unit vector in the $z$-direction, then the boundary conditions associated with the flow field are

$\mathbf{u}=U \mathbf{e}_{z} \quad$ on $\Omega_{\mathrm{p}}$,

$\mathbf{u}=0$ on $\Omega_{\mathrm{b}}$.

\subsection{Electrophoretic mobility}

For the present case, only the $z$-components of the forces acting on a particle, including the electrostatic force and the hydrodynamic force, need be considered. The $z$-component of the former, $F_{E z}$, can be calculated by integrating the Maxwell stress tensor over the particle surface,

$F_{E z}=\iint_{S}\left(\sigma^{E} \cdot \mathbf{n}\right) \cdot \mathbf{e}_{z} \mathrm{~d} S$,

where $S$ represents particle surface, $\sigma^{E}=\varepsilon \mathbf{E E}-(1 / 2) \varepsilon E^{2} \mathbf{I}$ is the Maxwell stress tensor, $\mathbf{E}=-\nabla \Psi=\mathbf{n}(\partial \Psi / \partial n)+\mathbf{t}(\partial \Psi / \partial t)$ is the applied electric field, $\mathbf{I}$ is the unit tensor, $\mathbf{t}$ is the unit tangential vector on the particle surface, $n$ and $t$ are, respectively, the magnitude of $\mathbf{n}$ and that of $\mathbf{t}$, and $E^{2}=\mathbf{E} \cdot \mathbf{E}$. Substituting $\sigma^{E}$ into Eq. (61) yields

$F_{E z}=\iint_{S}\left(\varepsilon \frac{\partial \Psi}{\partial n} \frac{\partial \Psi}{\partial z}-\frac{1}{2} \varepsilon\left[\left(\frac{\partial \Psi}{\partial n}\right)^{2}+\left(\frac{\partial \Psi}{\partial t}\right)^{2}\right] n_{z}\right) \mathrm{d} S$,

where $n_{z}$ is the $z$-component of $\mathbf{n}$. Since $\Psi=\Psi_{1}+\Psi_{2}$, this expression becomes

$$
\begin{aligned}
F_{E z}= & \iint_{S}\left(\varepsilon \frac{\partial \Psi_{1}}{\partial n} \frac{\partial \Psi_{1}}{\partial z}-\frac{1}{2} \varepsilon\left[\left(\frac{\partial \Psi_{1}}{\partial n}\right)^{2}+\left(\frac{\partial \Psi_{1}}{\partial t}\right)^{2}\right] n_{z}\right) \mathrm{d} S \\
& +\iint_{S}\left(\varepsilon \frac{\partial \Psi_{2}}{\partial n} \frac{\partial \Psi_{2}}{\partial z}-\frac{1}{2} \varepsilon\left[\left(\frac{\partial \Psi_{2}}{\partial n}\right)^{2}+\left(\frac{\partial \Psi_{2}}{\partial t}\right)^{2}\right] n_{z}\right) \mathrm{d} S \\
& +\iint_{S}\left(\varepsilon\left[\frac{\partial \Psi_{1}}{\partial n} \frac{\partial \Psi_{2}}{\partial z}+\frac{\partial \Psi_{2}}{\partial n} \frac{\partial \Psi_{1}}{\partial z}\right]\right. \\
& \left.-\frac{1}{2} \varepsilon\left[2 \frac{\partial \Psi_{1}}{\partial n} \frac{\partial \Psi_{2}}{\partial n}+2 \frac{\partial \Psi_{1}}{\partial t} \frac{\partial \Psi_{2}}{\partial t}\right] n_{z}\right) \mathrm{d} S .
\end{aligned}
$$

Because a particle cannot be driven by its equilibrium electrostatic field, the terms involving $\left(\partial \Psi_{1} / \partial n\right)^{2},\left(\partial \Psi_{1} / \partial t\right)^{2}$, and $\left(\partial \Psi_{1} / \partial n\right)\left(\partial \Psi_{1} / \partial z\right)$ should vanish. Also, $\left(\partial \Psi_{2} / \partial n\right)^{2},\left(\partial \Psi_{2} /\right.$ $\partial t)^{2}$, and $\left(\partial \Psi_{2} / \partial n\right)\left(\partial \Psi_{2} / \partial z\right)$ are all small. The magnitude of each of the rest terms is on the order of $E$, and therefore, Eq. (63) can be approximated by

$$
\begin{aligned}
F_{E z}= & \iint_{S}\left(\varepsilon\left[\frac{\partial \Psi_{1}}{\partial n} \frac{\partial \Psi_{2}}{\partial z}+\frac{\partial \Psi_{2}}{\partial n} \frac{\partial \Psi_{1}}{\partial z}\right]\right. \\
& \left.-\varepsilon\left[\frac{\partial \Psi_{1}}{\partial n} \frac{\partial \Psi_{2}}{\partial n}+\frac{\partial \Psi_{1}}{\partial t} \frac{\partial \Psi_{2}}{\partial t}\right] n_{z}\right) \mathrm{d} S .
\end{aligned}
$$


This expression is applicable to both symmetric and asymmetric systems at an arbitrary surface potential subject to all three types of surface conditions.

For a nonconductive particle, $\partial \Psi_{2} / \partial n=0$, and Eq. (64) becomes

$F_{E z}=\iint_{S}\left(\varepsilon\left[\frac{\partial \Psi_{1}}{\partial n} \frac{\partial \Psi_{2}}{\partial z}\right]-\varepsilon\left[\frac{\partial \Psi_{1}}{\partial t} \frac{\partial \Psi_{2}}{\partial t}\right] n_{z}\right) \mathrm{d} S$.

If the surface of a particle is maintained at a constant potential, $\partial \Psi_{1} / \partial t=0$, and Eq. (65) can further be simplified as

$F_{E z}=\iint_{S} \varepsilon \frac{\partial \Psi_{1}}{\partial n} \frac{\partial \Psi_{2}}{\partial z} \mathrm{~d} S=\iint_{S} \sigma E_{z} \mathrm{~d} S$

where $\sigma=-\varepsilon \mathbf{n} \cdot \nabla \Psi_{1}=-\varepsilon\left(\partial \Psi_{1} / \partial n\right)$ is the surface charge density, and $E_{z}=-\partial \Psi_{2} / \partial z$ is the strength of the local electric field in the $z$-direction. Equation (66) was used by Shugai and Carnie [13] for the electrophoresis of a sphere with a thick double layer parallel to a plane, normal to a plane, and along the axis of a cylindrical pore, and by $\mathrm{Hsu}$ and $\mathrm{Ku}$ [17] for the electrophoresis of a finite cylinder along the axis of a cylindrical pore. Both assumed a weak $\mathbf{E}$ and low, constant surface potential. It is interesting to note that for a symmetric system, since $\partial \Psi_{1} / \partial t=0$, Eq. (66) is not limited to the case of constant surface potential, and becomes applicable to all three types of surface conditions in this study. The electrophoresis of a finite cylinder along the axis of a cylindrical pore [15], for example, belongs to this category.

The expression

$F_{E z}=\iint_{S} \sigma E_{z} \mathrm{~d} S=\iint_{S} \sigma\left(-\frac{\partial \Psi}{\partial z}\right) \mathrm{d} S$

is widely used to evaluate $F_{E z}[10,16,19-21,23,24,29]$, where $\sigma=-\varepsilon \mathbf{n} \cdot \nabla \Psi=-\varepsilon(\partial \Psi / \partial n)$ is the surface charge density. In terms of $\Psi_{1}$ and $\Psi_{2}$, we have

$$
\begin{aligned}
F_{E z}= & \iint_{S}\left(\varepsilon \frac{\partial \Psi_{1}}{\partial n} \frac{\partial \Psi_{1}}{\partial z}+\varepsilon \frac{\partial \Psi_{1}}{\partial n} \frac{\partial \Psi_{2}}{\partial z}+\varepsilon \frac{\partial \Psi_{2}}{\partial n} \frac{\partial \Psi_{1}}{\partial z}\right. \\
& \left.+\varepsilon \frac{\partial \Psi_{2}}{\partial n} \frac{\partial \Psi_{2}}{\partial z}\right) \mathrm{d} S .
\end{aligned}
$$

The presence of the first, the third, and the fourth terms on the right-hand side of this expression implies that it is only applicable to some special symmetric electrophoresis problems such as a sphere moving at the center of a spherical cavity [19] or a sphere [20] or a spheroid [23] moving along the axis of a cylindrical pore.

The hydrodynamic force acting on a particle in the $z$-direction, $F_{D z}$, can be calculated by integrating the hydrodynamic stress tensor over its surface; that is,

$F_{D z}=\iint_{S}\left(\sigma^{\mathrm{H}} \cdot \mathbf{n}\right) \cdot \mathbf{e}_{z} \mathrm{~d} S$,

where $\sigma^{H}=-p \mathbf{I}+2 \eta \Delta$ is the hydrodynamic stress tensor, $\Delta=\left[\nabla \mathbf{u}+(\nabla \mathbf{u})^{T}\right] / 2$ is the rate of deformation tensor, and the superscript $\mathbf{T}$ denotes matrix transpose. Note that Eq. (69) is suitable for both rigid $[8,13]$ and soft particles $[10,28,30]$. If we
Table 1

Summary of the present method for the calculation of the electrophoretic mobility of a colloidal particle under the condition of weak applied electric field

1. Types of particle: rigid or soft

2. Governing equations:

(1) High surface potential [25-28]

(a) Electric field Eqs. (22)-(24) or Eqs. (32)-(35)

(b) Flow field

Eqs. (25) and (26) or Eqs. (36) and (37)

(2) Low surface potential [12-15]

(a) Electric field

Eqs. (27) and (28) or Eqs. (38) and (39)

(b) Flow field

Eqs. (29) and (30) or Eqs. (40) and (41)

3. Types of boundary conditions for electric field: constant surface potential, constant surface charge density, chargeregulated surface

4. Electrostatic force in $z$-direction:

$$
\begin{aligned}
F_{E z}= & \iint_{S}\left(\varepsilon\left[\frac{\partial \Psi_{1}}{\partial n} \frac{\partial \Psi_{2}}{\partial z}+\frac{\partial \Psi_{2}}{\partial n} \frac{\partial \Psi_{1}}{\partial z}\right]\right. \\
& \left.-\varepsilon\left[\frac{\partial \Psi_{1}}{\partial n} \frac{\partial \Psi_{2}}{\partial n}+\frac{\partial \Psi_{1}}{\partial t} \frac{\partial \Psi_{2}}{\partial t}\right] n_{z}\right) \mathrm{d} S
\end{aligned}
$$

5. Hydrodynamic force in $z$-direction:

$$
F_{D z}=\iint_{S}\left[t_{z} \eta\left(\frac{\partial u_{n}}{\partial t}+\frac{\partial u_{t}}{\partial n}\right)+n_{z}\left(-p+2 \eta \frac{\partial u_{n}}{\partial n}\right)\right] \mathrm{d} S
$$

6. Force balance at steady state:

$$
F_{E z}+F_{D z}=0
$$

let $\mathbf{u}=\mathbf{n} u_{n}+\mathbf{t} u_{t}=\mathbf{n}(\mathbf{u} \cdot \mathbf{n})+\mathbf{t}(\mathbf{u} \cdot \mathbf{t})$, then Eq. (69) can be rewritten as

$$
F_{D z}=\iint_{S}\left[t_{z} \eta\left(\frac{\partial u_{n}}{\partial t}+\frac{\partial u_{t}}{\partial n}\right)+n_{z}\left(-p+2 \eta \frac{\partial u_{n}}{\partial n}\right)\right] \mathrm{d} S .
$$

For a rigid particle $\left(\partial u_{n} / \partial n\right)_{S}=0$ and $\left(\partial u_{n} / \partial t\right)_{S}=0$; this expression can be further simplified as

$$
F_{D z}=\iint_{S} \eta \frac{\partial(\mathbf{u} \cdot \mathbf{t})}{\partial n} t_{z} \mathrm{~d} S+\iint_{S}-p n_{z} \mathrm{~d} S .
$$

Note that $F_{D z}$ comprises a viscous force and a pressure term [14-17,20,21,23,24,31]. The electrophoretic mobility of a particle can be determined from the fact that the net force acting on it in the $z$-direction vanishes at steady state; that is,

$F_{E z}+F_{D z}=0$.

\section{Conclusions}

The method proposed in this study is summarized in Table 1 , and two methods commonly used in the literature for the case of low surface potential are summarized in Table 2 . Since the present method is applicable to all types of electrophoresis problems, regardless of the surface conditions on a particle and the geometry of a system, it is the most general one among the three methods. As presented in Table 2, for an 
Table 2

Summary of two widely used methods for the calculation of the electrophoretic mobility of a colloidal particle under conditions of weak applied electric field and low surface potential

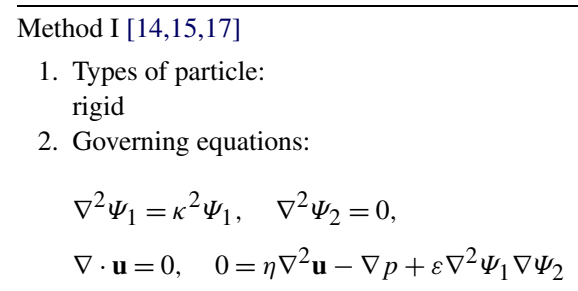

3. Types of boundary conditions for electric field:

(1) Arbitrary geometry constant surface potential

(2) Symmetry geometry constant surface potential, constant surface charge density, chargeregulated model

4. Electrostatic force in $z$-direction:

$$
F_{E z}=\iint_{S} \varepsilon \frac{\partial \Psi_{1}}{\partial n} \frac{\partial \Psi_{2}}{\partial z} \mathrm{~d} S=\iint_{S} \sigma E_{z} \mathrm{~d} S
$$

5. Hydrodynamic force in $z$-direction:

$$
F_{D z}=\iint_{S} \eta \frac{\partial(\mathbf{u} \cdot \mathbf{t})}{\partial n} t_{z} \mathrm{~d} S+\iint_{S}-p n_{z} \mathrm{~d} S
$$

6. Force balance at steady state:

$$
F_{E z}+F_{D z}=0
$$

Method II [16,19,20,23,24]

1. Types of particle: rigid

2. Governing equations:

$$
\begin{aligned}
& \nabla^{2} \Psi_{1}=\kappa^{2} \Psi_{1}, \quad \nabla^{2} \Psi_{2}=0 \\
& \nabla \cdot \mathbf{u}=0, \quad 0=\eta \nabla^{2} \mathbf{u}-\nabla p+\varepsilon \nabla^{2} \Psi \nabla \Psi
\end{aligned}
$$

3. Types of boundary conditions for electric field:

(1) Arbitrary geometry inapplicable, in general

(2) Symmetry geometry constant surface potential, constant surface charge density, chargeregulated model

4. Electrostatic force in $z$-direction

$$
F_{E z}=\iint_{S} \varepsilon \frac{\partial \Psi}{\partial n} \frac{\partial \Psi}{\partial z} \mathrm{~d} S=\iint_{S} \sigma E_{z} \mathrm{~d} S
$$

5. Hydrodynamic force in $z$-direction:

$$
F_{D z}=\iint_{S} \eta \frac{\partial(\mathbf{u} \cdot \mathbf{t})}{\partial n} t_{z} \mathrm{~d} S+\iint_{S}-p n_{z} \mathrm{~d} S
$$

6. Force balance at steady state:

$$
F_{E z}+F_{D z}=0
$$

arbitrary geometry, Method I is suitable for the electrophoresis of a rigid particle with a constant surface potential. For a symmetric geometry, it becomes applicable to all three types of boundary conditions. Method II is inapplicable to a problem of asymmetric nature, in general. It can be used for all three types of boundary conditions, however, if a problem is of symmetric nature.

\section{Acknowledgment}

This work is supported by the National Science Council of the Republic of China.

\section{References}

[1] E.H. Cao, R. Bryant, D.J.A. Williams, J. Colloid Interface Sci. 179 (1996) 143.

[2] M.J. Hernaiz, L.A. LeBrun, Y. Wu, J.W. Sen, R.J. Linhardt, N.H.H. Heegaard, Eur. J. Biochem. 269 (2002) 2860.

[3] S.R. Bean, G.L. Lookhart, Electrophoresis 19 (1998) 3190.

[4] M. Hajduch, R. Rakwal, G.K. Agrawal, M. Yonekura, A. Pretova, Electrophoresis 22 (2001) 2824.

[5] M. Von Smoluchowski, Z. Phys. Chem. 92 (1918) 129.

[6] E. Lee, F.Y. Yen, J.P. Hsu, Electrophoresis 21 (2000) 475.

[7] J.P. Hsu, E. Lee, F.Y. Yen, J. Chem. Phys. 112 (2000) 6404.

[8] A.A. Shugai, S.L. Carnie, D.Y.C. Chan, J.L. Anderson, J. Colloid Interface Sci. 191 (1997) 357.

[9] R.W. O'Brien, L.R. White, J. Chem. Soc. Faraday Trans. 2 (74) (1978) 1607.

[10] H. Ohshima, Adv. Colloid Interface Sci. 62 (1995) 189.

[11] D.C. Henry, Proc. R. Soc. London Ser. A 133 (1931) 106.

[12] J. Ennis, J.L. Anderson, J. Colloid Interface Sci. 185 (1997) 497.

[13] A.A. Shugai, S.L. Carnie, J. Colloid Interface Sci. 213 (1999) 298.

[14] J.P. Hsu, M.H. Ku, C.C. Kuo, Langmuir 21 (2005) 7588.

[15] J.P. Hsu, C.Y. Kao, J. Phys. Chem. B 106 (2002) 10605.

[16] J.P. Hsu, S.H. Hung, C.Y. Kao, Langmuir 18 (2002) 8897.

[17] J.P. Hsu, M.H. Ku, J. Colloid Interface Sci. 283 (2005) 592.

[18] A.L. Zydney, J. Colloid Interface Sci. 169 (1995) 476.

[19] E. Lee, J.W. Chu, J.P. Hsu, J. Colloid Interface Sci. 196 (1997) 316.

[20] J.P. Hsu, M.H. Ku, C.Y. Kao, J. Colloid Interface Sci. 276 (2004) 248.

[21] J.P. Hsu, M.H. Ku, C.Y. Kao, Ind. Eng. Chem. Res. 44 (2005) 1105.

[22] Y.P. Tang, M.H. Chih, E. Lee, J.P. Hsu, J. Colloid Interface Sci. 242 (2001) 121.

[23] J.P. Hsu, S.H. Hung, J. Colloid Interface Sci. 264 (2003) 121.

[24] H.Y. Yu, S.H. Hung, J.P. Hsu, Colloid Polym. Sci. 283 (2004) 10.

[25] M.H. Chih, E. Lee, J.P. Hsu, J. Colloid Interface Sci. 248 (2002) 383.

[26] E. Lee, J.W. Chu, J.P. Hsu, J. Colloid Interface Sci. 205 (1998) 65.

[27] J.W. Chu, W.H. Lin, E. Lee, J.P. Hsu, Langmuir 17 (2001) 6289.

[28] E. Lee, Y.P. Tang, J.P. Hsu, Langmuir 20 (2004) 9415.

[29] J.H. Masliyah, Electrokinetic Transport Phenomena, Alberta Oil Sands Technology and Research Authority, Edmonton, 1994.

[30] H. Ohshima, J. Colloid Interface Sci. 218 (1999) 535.

[31] G. Backstrom, Fluid Dynamics by Finite Element Analysis, Studentlitteratur, Lund, 1999. 